\title{
A Novel Two-Stage Thresholding Method for Segmentation of Malaria Parasites in Microscopic Blood Images
}

\author{
${ }^{1} \mathrm{~J}$. Somasekar, ${ }^{2}$ B.Eswara Reddy \\ ${ }^{1}$ Department of Computer Science and Engineering, Gopalan college of Engineering and Management, \\ Bangalore, India; \\ ${ }^{2}$ Professor of CSE and Principal, JNTUA College of Engineering, Kalikiri, \\ Andhra Pradesh, India; \\ 1.jsomasekar@gmail.com; ${ }^{2}$ principal.cek@jntua.ac.in
}

\begin{abstract}
Developing computerized diagnostic tool for the detection of malaria infected cells in microscopic blood images can help to reduce malaria-induced mortality. Segmentation of malaria infected cells is a key step in the automated malaria diagnosis pipeline. In this paper, a novel two-stage thresholding method for segmentation of malaria parasites in microscopic blood images for diagnosis is presented. The RGB microscopic image is converted into YUV color space and luminance component is considered for single channel processing. The infected parasites are segmented by the proposed threshold method, which is carried out in two stages by maximizing between-class variance of an original image and consequently by an iterative threshold selection from a stage-one threshold image with suitable stopping criteria. The experimental results on benchmark dataset that comprise more than 300 images show that the proposed method successfully detects malaria parasites with no prior knowledge of the contents of the image without parameter tuning.
\end{abstract}

Keywords: Microscopic Imaging; Malaria; Segmentation; Thresholding; Computerized Diagnosis.

\section{Introduction}

Malaria is a critical healthcare problem worldwide. According to the world malaria report of 2014 published by WHO, an estimated 3.3 billion people across the world are at risk of malaria, of whom 1.2 billion are at high risk and has led to 584,000 deaths [1]. Most deaths due to Malaria are of infants and children. The conventional method of diagnosing malaria depends on the microscopic examination of Giemsa-stained blood smears and this technique is considered the gold standard. Approximately, a total of 197 million patients worldwide are tested for malaria by microscopic examination. However, the manual method of diagnosis is tedious, requires expert technicians, and is prone to human erroneous; therefore it will affect the accuracy of the diagnosis (increasing false diagnosis). There are several studies suggesting a computer vision approach for the diagnosis of malaria through microscopic blood smear images. A detailed survey for segmentation of malaria parasites through microscopic imaging found in [2] [3]. Otsu or histogram thresholding has been attempted for malaria infected erythrocyte segmentation 
and It has limitation where textural variation is high [3]. Besides, it will not work for both uni-modal and bi-modal histogram images. Considering these problems, there is need for an automated system for diagnosis of malaria. Hence, we propose a threshold technique by which we can extract the infected cells and consequently detect infected cells in original images. The proposed thresholding technique is carried out in two stages. The first stage comprised maximizing between-class variance of a luminance component of an original color image and the optimal threshold value is calculated in stage-two by iterative selection algorithm. The rest of the article is organized as follows. The materials and methods for malaria parasite border detection is described in section 2. Section 3 presents the experimental results followed by conclusions are drawn in section 4.

\section{Materials and Methods}

\subsection{Dataset and Experiment processing}

The proposed method was performed using MATLAB 7.10.0 (R2010a) Programming software on a personal computer with an AMD Phenom II N830 triple-core processor $2.10 \mathrm{GHz}, 3 \mathrm{~GB}$ system memory and 64-bit Windows-7 operating system. The images from the DPDx database [http://www.cdc.gov/dpdx/malaria/gallery.html] were used for the experiments. Few more malaria images were supplied by Dr. Ashok K. Maiti, Department of Pathology, Midnapur Medical College \& Hospital, Midnapur, West Bengal, India, for testing of our proposed method.

\subsection{Methodology}

The detection of malaria infected cells through microscopic thin blood smear images consists of the following steps sequentially, (i) Preprocessing [5], (ii) stage-one threshold (initial threshold image) (iii) stage-two threshold, (iv) Parasite segmentation, and (v) post processing [5]. The following sub sections comprises in details of the steps involved.

The input color blood image be represented by

$$
[f(x, y)]_{m \times n}=\left[\begin{array}{l}
f_{R}(x, y) \\
f_{G}(x, y) \\
f_{B}(x, y)
\end{array}\right]_{m \times n}
$$

Where $R(x, y), G(x, y)$, and $B(x, y)$ denote the red, green, and blue color values of a pixel located at $(x, y)$ with $x, y$ are pixel coordinates in the width and height dimensions, respectively such that $1 \leq x \leq m$ and $1 \leq y \leq n[4]$.

\subsubsection{Preprocessing}

In this step, if the input image is RGB, it must be converted into YUV color space by using the following mathematical equations.

$$
\begin{gathered}
Y(x, y)=\left[\begin{array}{lll}
W_{R} & W_{G} & W_{B}
\end{array}\right] \times\left[f_{R}(x, y) f_{G}(x, y) f_{B}(x, y)\right]^{T} \\
U(x, y)=U_{\max }\left(\frac{f_{B}(x, y)-Y(x, y)}{1-W_{B}}\right)
\end{gathered}
$$


J. Somasekar, B. Eswara Reddy; A Novel Two-Stage Thresholding Method for Segmentation of Malaria Parasites in Microscopic Blood Images. Journal of Biomedical Engineering and Medical Imaging, Volume 4, No 2, April (2017), pp 31-42

$$
V(x, y)=V_{\max }\left(\frac{f_{R}(x, y)-Y(x, y)}{1-W_{R}}\right)
$$

Where, weighted values of $R, G, B$ are denoted by $W_{R}, W_{G}$ and $W_{B}$, these values are $0.299,0.587$ and 0.114 , respectively [6] [7]. The values of $U_{\max }$ and $V_{\max }$ are 0.436 and 0.615 , respectively. Equivalently, the matrix form for converting RGB to YUV color space as shown in Equation (5). In our method, the luminance component $(Y(x, y))$ is considered for further processing. The proposed method works in other color spaces namely RGB, HSI etc, but the main difference is, YUV color space takes less execution when compared to RGB and HIS.

$$
\left[\begin{array}{l}
Y(x, y) \\
U(x, y) \\
V(x, y)
\end{array}\right]=\left[\begin{array}{ccc}
0.299 & 0.587 & 0.114 \\
-0.147 & -0.288 & 0.436 \\
0.615 & -0.514 & -0.100
\end{array}\right]\left[\begin{array}{l}
f_{R}(x, y) \\
f_{G}(x, y) \\
f_{B}(x, y)
\end{array}\right]
$$

Median filter is applied in order to reduce noise in preprocessing step. A standard median operation is implemented by sliding a window of odd size over an image [8]. In the proposed method window of size $3 \times 3$ is used for median filter. The mathematical equation for median filter is shown in Equation (6).

$$
y[m, n]=\operatorname{median}\{x[i, j]:(i, j) \in W\}
$$

Where $W$ represents a neighborhood defined by the user, centered around location $[m, n]$ in the image.

\subsubsection{Stage-one thresholding}

The Heaviside function $U(t)$ of a single variable $t$ is defined by the following equation.

$$
\mathrm{U}(\mathrm{t})=\left\{\begin{array}{l}
1, \text { if } \mathrm{t} \geq 0 \\
0, \text { elsewhere }
\end{array}\right.
$$

The major mathematical equation of the initial threshold image (stage-one threshold) for aiming to remove few pixels which are not infected with malaria parasites in luminance component $\mathrm{Y}(\mathrm{x}, \mathrm{y})$ of size $\mathrm{m} \times \mathrm{n}$, is provided as follows.

$$
\mathrm{f}(\mathrm{x}, \mathrm{y})=\mathrm{Y}(\mathrm{x}, \mathrm{y})[\mathrm{U}(\lambda-\mathrm{Y}(\mathrm{x}, \mathrm{y}))]+\alpha[\mathrm{U}(\mathrm{Y}(\mathrm{x}, \mathrm{y})-\lambda)]
$$

Where $\mathrm{U}($.$) is a Heaviside function as defined in the Equation (7), and \lambda, \alpha$ are the parameters. The value of the parameter $\alpha$ is set to be $(L-1) \times 0.7$ with $L$ represents number bins used in the histogram of an image $Y(x, y)$. The selection $\alpha$ will be discussed in the experimental section. The selection of $\lambda$ is calculated as follows. Define the between-class variance $V_{b}(t)$ by using the within-class variance $V_{w}(t)$ as shown below [8].

$$
\begin{aligned}
V_{b}(t)=V(t) & -V_{w}(t) \\
= & V(t)-\left[W_{1}(t) V_{1}(t)+W_{2}(t) V_{2}(t)\right] \\
= & W_{1}(t)\left[\mu_{1}(t)-\mu\right]^{2}+W_{2}(t)\left[\mu_{2}(t)-\mu\right]^{2} \\
= & W_{1}(t) W_{2}(t)\left[\mu_{1}(t)-\mu_{2}(t)\right]^{2}
\end{aligned}
$$

where $\mathrm{W}_{1}(\mathrm{t}), \mathrm{V}_{1}(\mathrm{t})$ and $\mu_{1}(\mathrm{t})$ indicate total amount, variance and the mean of inside class, respectively, as shown in equations (10)-(12).

$$
\mathrm{W}_{1}=\sum \mathrm{p}(\mathrm{i})
$$




$$
\begin{gathered}
\mathrm{V}_{1}=\sum \mathrm{p}(\mathrm{i})\left[\mathrm{x}(\mathrm{i})-\mu_{1}\right]^{2} / \mathrm{W}_{1} \\
\mu_{1}=\sum \mathrm{p}(\mathrm{i}) \mathrm{x}(\mathrm{i}) / \mathrm{W}_{1}
\end{gathered}
$$

Analogous definition can be provided for the corresponding values $W_{2}(t), V_{2}(t)$ and $\mu_{2}(t)$ of the between-class variance [8]. Thus the value of the parameter $\lambda$ can be calculated by using the Equation (13):

$$
(\mathrm{L}-1) \times \underbrace{\operatorname{Arg} \max }_{0 \leq \mathrm{t} \leq \mathrm{N}-1}\left\{\mathrm{~W}_{1}(\mathrm{t}) \mathrm{W}_{2}(\mathrm{t})\left[\mu_{1}(\mathrm{t})-\mu_{2}(\mathrm{t})\right]^{2}\right\}
$$

\subsubsection{Stage-two thresholding}

In a stage-two threshold image, we aim to find an optimal threshold value by which we can extract the parasite infected cells. However, it is not easy to fix a threshold value because malaria microscopic blood images are heterogeneous. Hence, we developed an iterative threshold selection algorithm with a suitable terminating factor for finding an optimal threshold value for parasite extraction using a stage-one threshold image $\mathrm{f}(\mathrm{x}, \mathrm{y})$ as an input image.

The detection of the infected cells can be modeled by the following classification problem. For a stageone threshold image $f(x, y)$, there are pixels that belong to two classes: $G_{1}$ (infected ones) and $G_{2}$ (the background). The segmentation of the infected cells then becomes a problem for choosing one null hypothesis $\left(\mathrm{H}_{0}\right)$ and an alternative hypothesis $\left(\mathrm{H}_{1}\right)$ :

$$
\begin{aligned}
& \mathrm{H}_{0}:[\mathrm{f}(\mathrm{x}, \mathrm{y})] \in \mathrm{G}_{1} \\
& \mathrm{H}_{1}:[\mathrm{f}(\mathrm{x}, \mathrm{y})] \in \mathrm{G}_{2}
\end{aligned}
$$

The above classification can be solved by using the initial threshold. In a general mathematical sense, a histogram of an image $f(x, y)$ with intensity levels in the range $[0, L-1]$ is a function as shown in Equation (14) and conforms to the condition shown in Equation (15).

$$
\begin{aligned}
H(k)= & \sum_{t \in T} \delta\left(X_{t}-k\right) \\
& \sum_{k=0}^{L-1} H(k)=m n
\end{aligned}
$$

Therefore, $H(k)$ computes the number of pixels that take on the value $k$. The two extreme points of a state-one threshold image can be calculated by using a histogram that is shown in Equation (14) and by using Equation (16).

$$
\left[\begin{array}{l}
f^{m}(x, y) \\
f^{M}(x, y)
\end{array}\right]=\left[\begin{array}{c}
\underset{k}{\operatorname{Arg} \operatorname{Min}}\{H(k)>0\} \\
\underset{k}{\operatorname{Arg} \operatorname{Max}}\{k H(k)>0\}
\end{array}\right]
$$

The initial threshold value $\mathrm{T}_{0}$ is calculated with a constant $\psi=0.5$ by using Equation (17).

$$
\begin{aligned}
\mathrm{T}_{0} & =\psi\left[f^{M}(x, y)+f^{m}(x, y)\right]+\left|\left(\frac{\sigma_{1}}{f^{M}(x, y)-f^{m}(x, y)}\right) \ln (\sigma)-\left(\frac{\sigma_{1}}{f^{M}(x, y)-f^{m}(x, y)}\right) \ln \left(\sigma_{1}\right)\right| \\
& =\psi\left[f^{M}(x, y)+f^{m}(x, y)\right]+\left|\left(\frac{\sigma_{1}}{f^{M}(x, y)-f^{m}(x, y)}\right)\left(\ln (\sigma)-\ln \left(\sigma_{1}\right)\right)\right|
\end{aligned}
$$




$$
=\psi\left[f^{M}(x, y)+f^{m}(x, y)\right]+\left|\left(\frac{\sigma_{1}}{f^{M}(x, y)-f^{m}(x, y)}\right)\left[\ln \left(\frac{\sigma}{\sigma_{1}}\right)\right]\right|
$$

In Eqn. (17), the quantities $\sigma, \sigma_{1}, \mu$ and $\mu_{1}$ are evaluated by using equations (18) to (21), respectively.

$$
\begin{gathered}
\sigma=\left[\frac{1}{m n-1} \sum_{x} \sum_{y}(h(x, y)-\mu)^{2}\right]^{\psi} \\
\sigma_{1}=\left[\frac{1}{m n-1} \sum_{x} \sum_{y}\left(f(x, y)-\mu_{1}\right)^{2}\right]^{\psi} \\
\mu=\frac{1}{m n} \sum_{x} \sum_{y} h(x, y) \\
\mu_{1}=\frac{1}{m n} \sum_{x} \sum_{y} f(x, y)
\end{gathered}
$$

The image is divided into two classes $\mathrm{G}_{1}$ and $\mathrm{G}_{2}$ based on the initial threshold value. The mathematical expressions for two classes $G_{i}(i=1,2)$ are as follows.

$$
\left\{\begin{array}{l}
f(x, y) \in G_{1}: f(x, y)>T_{0} \\
f(x, y) \in G_{2}: f(x, y)<T_{0} \\
f(x, y) \in G_{1}: f(x, y)=T_{0}
\end{array}\right.
$$

If $G_{1}$ and $G_{2}$ are the normalized foreground and backgrounds, then the histogram is the sum of these distributions $\mathrm{f}(\mathrm{z})$ and $\mathrm{g}(\mathrm{z}), \mathrm{z}$ is the gray level. Define the Gaussian distributions of two classes $\mathrm{G}_{1}$ and $\mathrm{G}_{2}$ are $\left\{f(z): z \sim N\left(\mu_{1}, \sigma_{1}\right)\right\}$ and $\left\{g(z): z \sim N\left(\mu_{2}, \sigma_{2}\right)\right\}$. The updated threshold value $\mathrm{T}$ is calculated by making as assumption that the Gaussian distribution of two classes $\mathrm{G}_{1}$ and $\mathrm{G}_{2}$, is parallel and will be given by

$$
\begin{gathered}
\frac{1}{\sigma_{1} \sqrt{2 \Pi}} \exp \left(\frac{-\left(\mathrm{T}-\mu_{1}\right)^{2}}{2 \sigma_{1}{ }^{2}}\right)=\frac{1}{\sigma_{2} \sqrt{2 \Pi}} \exp \left(\frac{-\left(\mathrm{T}-\mu_{2}\right)^{2}}{2 \sigma_{2}^{2}}\right) \\
\ln \left(\frac{1}{\sigma_{1}} \exp \left(\frac{-\left(\mathrm{T}-\mu_{1}\right)^{2}}{2 \sigma_{1}{ }^{2}}\right)\right)=\ln \left(\frac{1}{\sigma_{2}} \exp \left(\frac{-\left(\mathrm{T}-\mu_{2}\right)^{2}}{2 \sigma_{2}^{2}}\right)\right) \\
-\ln \left(\sigma_{1}\right)-\frac{\left(\mathrm{T}-\mu_{1}\right)^{2}}{2 \sigma_{1}{ }^{2}}=-\ln \left(\sigma_{2}\right)-\frac{\left(\mathrm{T}-\mu_{2}\right)^{2}}{2 \sigma_{2}^{2}} \\
\ln \left(\frac{\sigma_{2}}{\sigma_{1}}\right)+\frac{\left(\mathrm{T}-\mu_{2}\right)^{2}}{2 \sigma_{2}^{2}}-\frac{\left(\mathrm{T}-\mu_{1}\right)^{2}}{2 \sigma_{1}{ }^{2}}=0
\end{gathered}
$$




$$
\begin{gathered}
\ln \left(\frac{\sigma_{2}}{\sigma_{1}}\right)+\frac{\mathrm{T}^{2}+\mu_{2}{ }^{2}-2 \mathrm{~T} \mu_{2}}{2 \sigma_{2}^{2}}-\frac{\mathrm{T}^{2}+\mu_{1}{ }^{2}-2 \mathrm{~T} \mu_{1}}{2 \sigma_{1}{ }^{2}}=0 \\
\frac{1}{2 \sigma_{1}{ }^{2}{\sigma_{2}}^{2}}\left[\mathrm{~T}^{2}\left({\sigma_{2}}^{2}-{\sigma_{1}}^{2}\right)-2 \mathrm{~T}\left({\sigma_{1}}^{2} \mu_{2}-\mu_{1}{\sigma_{2}}^{2}\right)+\mu_{2}{ }^{2}{\sigma_{1}}^{2}-\mu_{1}{ }^{2}{\sigma_{2}}^{2}\right]+\ln \left(\frac{\sigma_{2}}{\sigma_{1}}\right)=0 \\
{\left[\mathrm{~T}^{2}\left({\sigma_{2}}^{2}-{\sigma_{1}}^{2}\right)-2 \mathrm{~T}\left({\sigma_{1}}^{2} \mu_{2}-\mu_{1}{\sigma_{2}}^{2}\right)+\mu_{2}{ }^{2}{\sigma_{1}}^{2}-\mu_{1}{ }^{2}{\sigma_{2}}^{2}\right]+2{\sigma_{1}}^{2} \sigma_{2}{ }^{2} \ln \left(\frac{\sigma_{2}}{\sigma_{1}}\right)=0}
\end{gathered}
$$

If the standard deviations of the two classes are coincides, then

$$
\begin{aligned}
2 \mathrm{~T}\left(\sigma^{2} \mu_{2}-\mu_{1} \sigma^{2}\right)+ & \mu_{2}{ }^{2} \sigma^{2}-\mu_{1}{ }^{2} \sigma^{2}=0 \\
& 2 \mathrm{~T}\left(\mu_{2}-\mu_{1}\right)+\mu_{2}{ }^{2}-\mu_{1}{ }^{2}=0
\end{aligned}
$$

Therefore, the updated threshold value is defined by

$$
\psi\left[\frac{1}{\mathrm{pq}} \sum_{\mathrm{x}=1}^{\mathrm{p}} \sum_{\mathrm{y}=1}^{\mathrm{q}} \mathrm{f}(\mathrm{x}, \mathrm{y})+\frac{1}{\mathrm{kl}} \sum_{\mathrm{x}=1}^{\mathrm{k}} \sum_{\mathrm{y}=1}^{\mathrm{l}} \mathrm{f}(\mathrm{x}, \mathrm{y})\right]
$$

Where, $1 \leq(\mathrm{p}, \mathrm{k}) \leq \mathrm{m}, 1 \leq(\mathrm{q}, \mathrm{s}) \leq \mathrm{n}$ s.t. $\mathrm{p}+\mathrm{k}=\mathrm{m}, \mathrm{q}+\mathrm{s}=\mathrm{n}$.

This can be expressed by using histogram of a stage-one threshold image as follows [9]:

$$
\psi\left\{\left[\sum_{\mathrm{k}=0}^{\mathrm{T}_{0}} \mathrm{kH}(\mathrm{k}) / \sum_{\mathrm{k}=0}^{\mathrm{T}_{0}} \mathrm{H}(\mathrm{k})\right]+\left[\left[\sum_{\mathrm{k}=1+\mathrm{T}_{0}}^{\mathrm{L}-1} \mathrm{kH}(\mathrm{k}) / \sum_{\mathrm{k}=1+\mathrm{T}_{0}}^{\mathrm{L}-1} \mathrm{H}(\mathrm{k})\right]\right]\right\}
$$

The optimal threshold value $\mathrm{T}_{\text {opt }}$ is obtained from Eqn. (24) such that it holds the following inequality.

$$
\lim _{\mathrm{k} \rightarrow \infty}\left|\mathrm{T}_{\mathrm{k}}-\mathrm{T}_{\mathrm{k}+1}\right|<\epsilon
$$

The value for terminating factor $\epsilon$ is set for the proposed work as 0.4 . The selection of threshold value by the proposed method is shown in Fig. 1 in which red dotted line indicates stage-one threshold value and green dotted line indicates optimal threshold value.

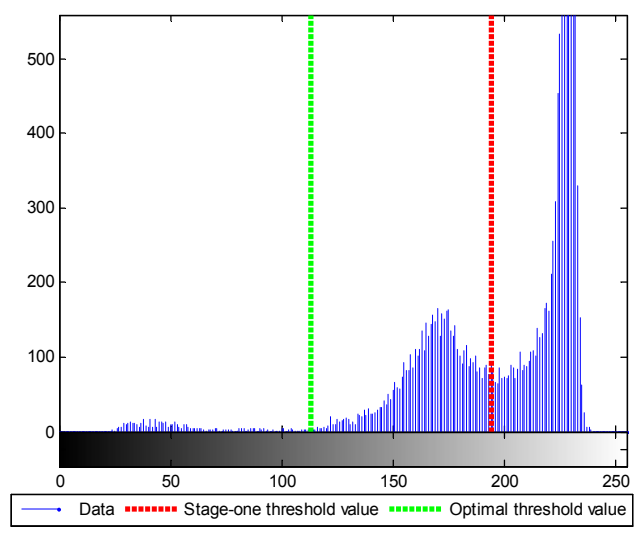

Figure. 1: Threshold selection for the test image for the proposed TSTM for malaria parasite segmentation. 
J. Somasekar, B. Eswara Reddy; A Novel Two-Stage Thresholding Method for Segmentation of Malaria Parasites in Microscopic Blood Images. Journal of Biomedical Engineering and Medical Imaging, Volume 4, No 2, April (2017), pp 31-42

\subsubsection{Malaria Parasite Segmentation}

The segmentation of malaria parasites is carried out for each element of the image, $h(x, y)$ using the optimal threshold value $T_{o p t}$ obtained in stage-two threshold. The malaria parasite detection image is obtained as follows.

$$
\begin{array}{r}
g(x, y)=W\left(h(x, y)-T_{\text {opt }}\right) \\
\text { s.t., } W(k)= \begin{cases}0, & k<0 \\
1, & k \geq 0\end{cases}
\end{array}
$$

Each value of an image $\mathrm{g}(\mathrm{x}, \mathrm{y})$ has either 0 or 1 . The elements of the image $\mathrm{g}(\mathrm{x}, \mathrm{y})$ with value $1($ white $)$ is belongs to the signal (infected one) otherwise normal.

\subsubsection{Post Processing}

Frequently, the segmented results are post processed to improve the accuracy of the detection of malaria parasites. In many cases, morphological operations are employed for this purpose. These operations are capable of filling the holes in parasites created in previous steps and eliminating all the elements in the image that are smaller than the structural element. In order to make the segmented object look better, the objects in the processed image can be smoothed by eroding the image. It removes outer layer of pixels yields more accurate enumeration results. If a black pixel has a white neighbor, then all the pixels are made white. The erosion of a binary image $A$ by the square structuring element $B$ with $3 \times 3$ can be expressed mathematically as [10] [11][12],

$$
A \ominus B=\left\{w: B_{w} \subseteq A\right\} \quad \text { where, } B_{w}=\{b+w: b \in B\}
$$

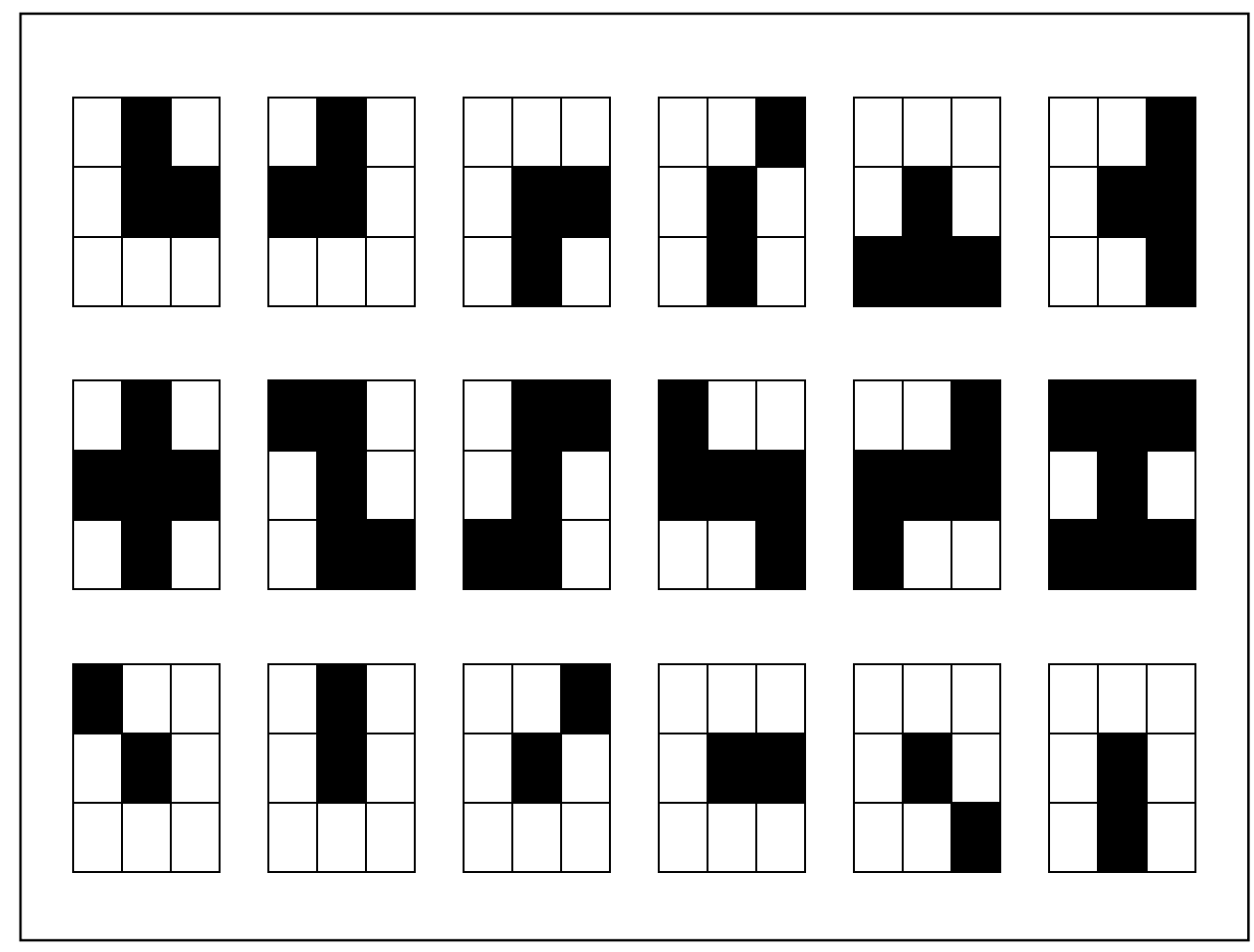

Figure.2: Different Masks used in the erosion operation. 
We selected square matrix structuring element of size $3 \times 3$. The selection of mask has a key role in achieving desired result. The erosion operation applied to our proposed method by using any of the $3 \times 3$ kernels as shown in Fig.2. The closing operation is applied to eroded image by using a square structuring element of size $5 \times 5$, which tends to smooth sections of contours and generally fuses narrow breaks and fills gaps in the contour [12]. The closing of $A$ by $B$ is the dilation of $A$ by $B$, followed by an erosion of the result by $B$.

\section{Experimental Results}

In order to quantitatively evaluate the performance of the proposed threshold method for malaria parasite detection by dice similarity coefficient (DSC) and relative foreground area error (RFAE) $[13,14]$. The detailed description of these measures is discussed in the following subsections.

\subsection{DSC}

It measures the overlap between the segmented image obtained by our proposed method and reference manual annotations. It is defined as the harmonic mean of Precision ( $P r)$ and Recall (Rc) and can be calculated as follows.

$$
\begin{gathered}
\operatorname{DSC}\left(M_{\text {seg }}, T_{\text {seg }}\right)=\frac{2}{\left(\frac{1}{P r}\right)+\left(\frac{1}{R c}\right)} \\
=\frac{2\left|M_{\text {seg }} \cap T_{\text {seg }}\right|}{\left(\frac{\left|M_{\text {seg }}\right|}{\left|M_{\text {seg }} \cap T_{\text {seg }}\right|}\right)+\left(\frac{\left|T_{\text {seg }}\right|}{\left|M_{\text {seg }} \cap T_{\text {seg }}\right|}\right)} \\
=\frac{2\left|M_{\text {seg }} \cap T_{\text {seg }}\right|}{\left|M_{\text {seg }}\right|+\left|T_{\text {seg }}\right|} \\
=\frac{\left|M_{\text {seg }} \cap T_{\text {seg }}\right|}{\left|M_{\text {seg }} \cap T_{\text {seg }}\right|+\frac{1}{2}\left|M_{\text {seg }} / T_{\text {seg }}\right|+\frac{1}{2}\left|T_{\text {seg }} / M_{\text {seg }}\right|}
\end{gathered}
$$

The DSC represents spatial overlap and has the following three properties. A DSC value more than 0.7 indicates excellent agreement.

Case (i): (no overlap)

If $M_{\text {seg }} \cap T_{\text {seg }}=0$, then $D S C=0$.

Case (ii): (partial overlap)

If $M_{\text {seg }} \cap T_{\text {seg }} \neq 0$ but then $0<D S C<1$.

Case (iii): (complete overlap)

If $\left[\left(M_{\text {seg }} \cap T_{\text {seg }}=T_{\text {seg }}\right) \wedge\left(M_{\text {seg }} \cap T_{\text {seg }}=M_{\text {seg }}\right)\right]=1$ then DSC $=1$. 
J. Somasekar, B. Eswara Reddy; A Novel Two-Stage Thresholding Method for Segmentation of Malaria Parasites in Microscopic Blood Images. Journal of Biomedical Engineering and Medical Imaging, Volume 4, No 2, April (2017), pp 31-42

The average DSC for the tested images using the proposed method is 0.8 . Hence the proposed thresholding method effectively detects the malaria infected cells.

\subsection{RFAE}

We also look at the object properties such as area and shape, as obtained from the segmented image by our method with respect to the manual annotation, is calculated by using relative foreground area error (RFAE). Hence, RFAE is calculated by using the following equation.

$$
\left\{\begin{array}{l}
\frac{A_{T}-A_{R}}{A_{T}} \quad \text { if } A_{T} \geq A_{R} \\
\frac{A_{R}-A_{T}}{A_{R}} \quad \text { if } A_{T}<A_{R}
\end{array}\right.
$$

Where $A_{T}$ is the area of segmented image and $A_{R}$ is the area of manual annotations. If RFAE is zero for perfect match of the segmented regions otherwise one. The smaller value of RFAE represents the higher accuracy. It can be observed that the parasite detection provided by the proposed thresholding method mean RFAE is 0.12 .

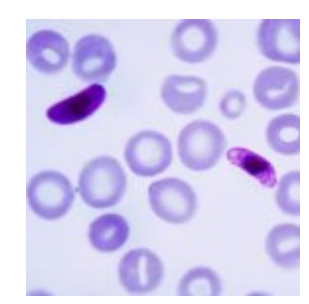

(a)

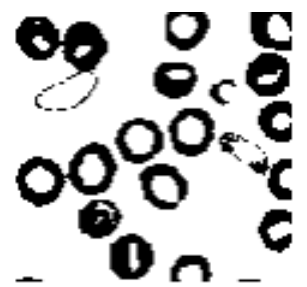

(e)

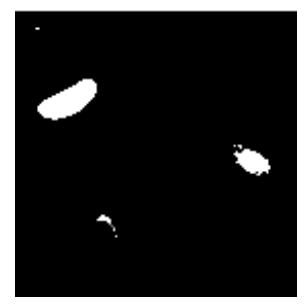

(i)

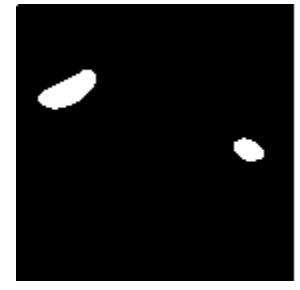

(b)

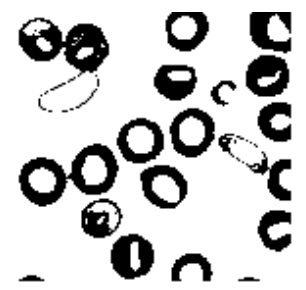

(f)

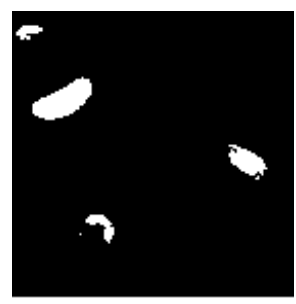

(j)

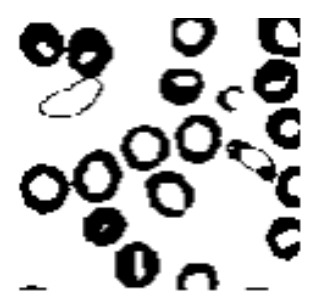

(c)

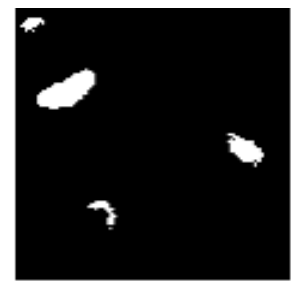

(g)

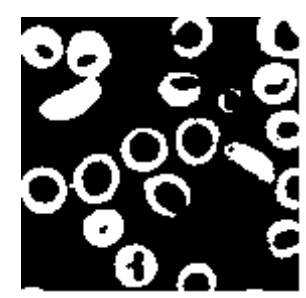

(k)

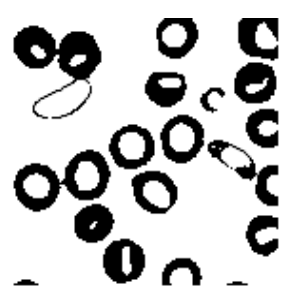

(d)

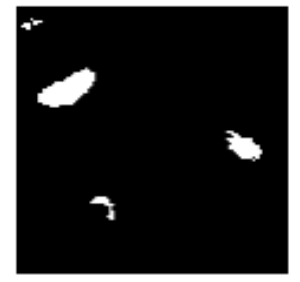

(h)

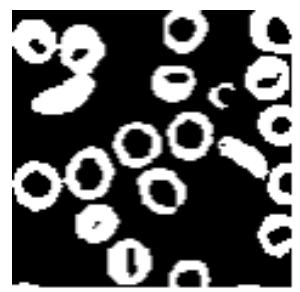

(1)

Figure.3: Segmentation results for various values of $\alpha$ for malaria microscopicy image: (a) Original, (b) ground truth image, (c)-(I) proposed thresholding results for different values of $\alpha$ with $\alpha=0.1,0.2,0.3,0.4$, $0.5,0.6,0.7,0.8,0.9$, and 1.0 , respectively. 


\subsection{Parameter selection}

In the proposed method, there are two parameters: $\alpha$ and $\lambda$. However, $\lambda$ can be automatically determined. Hence the parameter $\alpha$ is left uncertain and which assists to obtain the accurate final malaria parasite detection image. A series of experiements on microscopic malaria blood images with different values of $\alpha$ have been carried out. The value of $\alpha$ holds the Equation (35) and the maximum segmentation accuracy of the proposed method was obtained for $\alpha$ is $(L-1) * 0.7$ which was fixed for subsequent experiments.

$$
(L-1) * \underset{0<\alpha<1}{\operatorname{Arg} \min }\left[1-\left(\frac{\left|\Omega_{B} \cap \Omega_{T B}\right|+\left|\Omega_{F} \cap \Omega_{T F}\right|}{\left|\Omega_{B}\right|+\left|\Omega_{F}\right|}\right)\right]
$$

Where $\Omega_{T B}$ and $\Omega_{T F}$ denote the background and foreground area pixels in the thresholded image, $\Omega_{B}$ and $\Omega_{F}$ are the background and foreground of the manual annotations (ground truth image), and |. | is the cardinality of the set. The segmentation results for various values of $\alpha$ in the proposed method for malaria microscopic test image as shown in Figure 3.
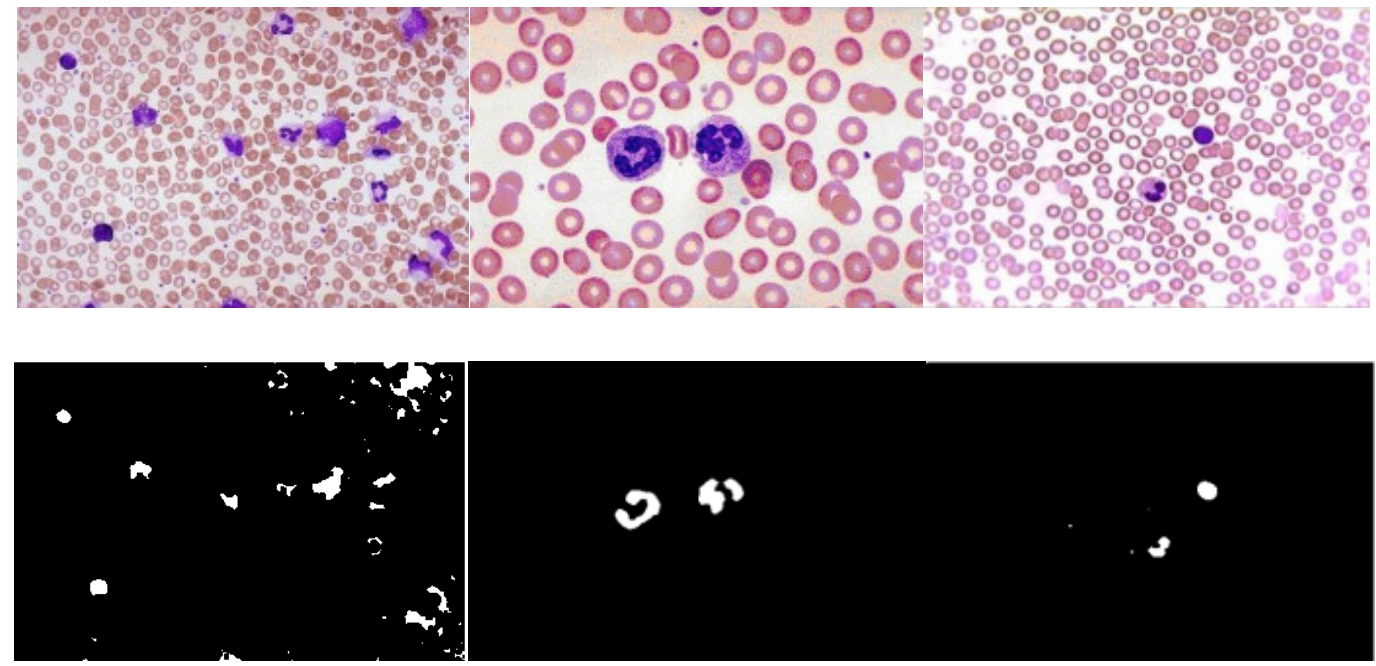

Figure.4: Segmentation of malaria parasite infected cells by proposed method: First row indicates original microscopic malaria infected images and second row represents malaria parasite infected cells extraction.
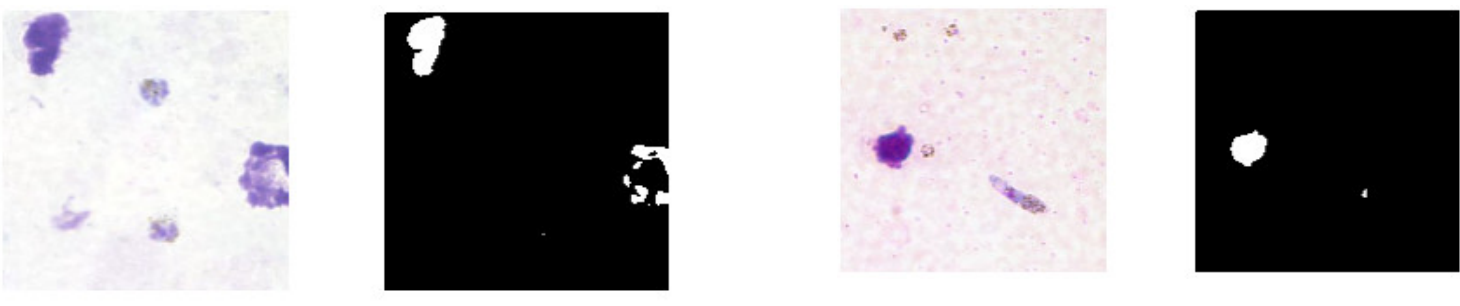

Figure. 5: Malaria parasites extraction results in thick blood smear images. 
J. Somasekar, B. Eswara Reddy; A Novel Two-Stage Thresholding Method for Segmentation of Malaria Parasites in Microscopic Blood Images. Journal of Biomedical Engineering and Medical Imaging, Volume 4, No 2, April (2017), pp 31-42

The malaria parasite detection results are illustrated for sample microscopic thin blood image as shown in Figure 4. To test the efficiency of our proposed method, we conducted experiments on thick blood smear images and segmentation results are shown in Figure 5. Hence proposed method works well for both thick and thin blood smear images. Besides, the proposed method applied for normal blood samples and works well.

\section{Conclusion}

In this paper, we have proposed a two-stage thresholding method for segmentation of malaria infected cells in microscopic blood images for assisting diagnosis. First, the colour image is converted in to into YUV color space for single channel processing. The infected parasites are detected by the proposed threshold method that comprises the maximizing the between-class variance of an original image and iterative threshold selection from stage-one image for segmentation of malaria parasites. The proposed method outperforms well based on the quantitative results DSC and RFAE. The work is useful in telepathology applications for computer aided diagnosis (CAD). The proposed method has ability to extract malaria infected cells in both thick and thin blood smear images.

\section{CONFLICTS OF INTEREST}

The authors have no conflict of interest.

\section{ACKNOWLEDGEMENTS}

We would like to thank Dr. Ashok K. Maiti, Department of Pathology, Midnapur Medical College \& Hospital, Midnapur, West Bengal, India, for providing microscopic blood images to test our proposed method. In addition, we would like to express our gratitude to Ching-Hao Lai, Taiwan for many helpful discussions. Moreover, we appreciate Zuoyong Li, Nanjing University of Science and Technology, Nanjing, China, for his guidance during creating manual annotation to find performance of our method.

\section{REFERENCES}

[1] WHO, World Malaria Report, Geneva, Switzerland 2015; 2-10.

[2] J.Somasekar, B.Eswara Reddy, Segmentation of erythrocytes infected with malaria parasites for the diagnosis using microscopy imaging, Computers and Electrical Engineering 2015; 45; 336-351.

[3] D.K. Das, R. Mukherjee, C. Chakraborty, Computational microscopic imaging for malaria parasite detection: a systematic review, Journal of Microscopy 2015; 260; 1-19.

[4] Yitian Zhao, Lavdie Rada, Ke Chen, Simon P. Harding, Yalin Zheng, Automated Vessel Segmentation Using Infinite Perimeter Active Contour Model with Hybrid Region Information with Application to Retinal Images, IEEE Transactions on Medical Imaging 2015; 34; 1797-1807.

[5] M.Emre Celebi, Hitoshi Iyatomi, Gerald Schaefer, William v.stoecker, Lession border detection in dermoscopy images, computerized medical imaging and graphics 2009; 33; 148-153. 
[6] Zaher Hamid Al-Tairi, Rahmita Wirza Rahmat, M. Iqbal Saripan, and Puteri Suhaiza Sulaiman, Skin Segmentation Using YUV and RGB Color Spaces, J Inf Process Systems 2014; 10; 283-299.

[7] Noor A. Ibraheem, Mokhtar M. Hasan, Rafiqul Z. Khan, Pramod K. Mishra, Understanding Color Models: A Review , ARPN Journal of Science and Technology 2012; 2; 265-275.

[8] Otsu, N., A Threshold Selection Method from Gray-Level Histograms, IEEE Transactions on Systems, Man, and Cybernetics $1979 ; 9 ; 62-66$.

[9] T.W. Ridler, S. Calvard, Picture thresholding using an iterative selection method, IEEE Trans. System, Man and Cybernetics 1978; 8; 630-632.

[10] R. C. Gonzalez, R. E. Woods, Digital image processing, second ed., Addison-Wesley, 1992.

[11] Rafael C.Gonzalez, Richard E.Woods, Steven L.Eddins, Digital Image Processing using MATLAB, second ed., Tata McGraw Hill Education Private Limited, New Delhi, 2011.

[12] Geoff Dougherty, Digital image processing for medical applications, First south Asian ed., Cambridge University press, New Delhi, 2010.

[13] Yitian Zhao, Lavdie Rada, Ke Chen, Simon P. Harding, and Yalin Zheng, Automated Vessel Segmentation Using Infinite Perimeter Active Contour Model with Hybrid Region Information with Application to Retinal Images, IEEE Transactions on Medical Imaging 2015; 34; 1797-1807.

[14] Mehmet Sezgin,Bulent Sankur, Survey over image thresholding techniques and quantitative performance evaluation, Journal of Electronic Imaging 2004; 13; 146-165. 